Pure Appl. Chem., Vol. 72, No. 9, pp. 1777-1781, 2000.

(C) 2000 IUPAC

\title{
Stereochemical support for Serratia endonuclease active-site geometry*
}

\author{
W. J. Stec ${ }^{1, \dagger}$, M. Koziolkiewicz ${ }^{1}$, and K. Taira ${ }^{2}$ \\ ${ }^{1}$ Department of Bioorganic Chemistry, Centre of Molecular and Macromolecular \\ Studies, Polish Academy of Sciences, 90-363 kódź, Poland; ${ }^{2}$ Department of \\ Chemistry and Biotechnology, Graduate School of Engineering, University of \\ Tokyo, Hongo, Tokyo 113-8656, Japan
}

\begin{abstract}
Earlier observation that stereoregular $A l l-R_{p}-P S$-oligonucleotides in the presence of nonsequence-specific Serratia endonuclease undergo nucleolytic degradation, confronted with recently published results on the active-site architecture of this enzyme, strongly supports an involvement of $3^{\prime}$-bridging and $\mathrm{pro}-\mathrm{Sp}$-nonbridging oxygen atoms of scissile internucleotide bond in the interactions with hydrated magnesium ion anchored by Asn-119 residue of this endonuclease.
\end{abstract}

Recently, the similarity between the active site of I-PpoI specific endonuclease and Serratia marcescens nonsequence-specific endonuclease was pointed out by two independent groups [1,2]. The different folding of these two proteins determines the specificity requirement, but both proteins contain conserved essential asparagine and histidine residues at their active sites and exhibit a similar one metalion co-ordination pattern. Therefore, all main active-site components required for the cleavage of the internucleotide phosphorus-oxygen bond are in the similar arrangement.

The general-base histidine 98 (I-PpoI) or 89 (Serratia) residue activates a water molecule for its nucleophilic attack at phosphorus and this occurs opposite from the site of the leaving 3'-oxygen. The ligand for the protein is comprised of the magnesium ion surrounded by three water molecules and Asn-119. Upon binding of the protein, the magnesium ion stabilizes the transition state by coordinating two oxygens of scissile phosphate, namely the 3'-oxygen of $\mathrm{P}-\mathrm{O}-\mathrm{C}^{3}$ bond and the pro-Sp-nonbridging oxygen (Fig. 1A; a) [3]. This magnesium ion does not coordinate a water molecule for nucleophilic attack at phosphorus opposite the leaving 3'-oxygen. Such architecture of the active site of the Serratia marcescens endonuclease comprising coordination of two oxygen atoms of the same scissile phosphate to the metal ion seems to be more plausible than the alternate mechanism for the mode of action of Serratia enzyme discussed by Miller et al. [4] and presented in Fig. 1B. The metal-bound water is deprotonated by a general base and attacks the phosphorus atom of the negatively charged phosphate, coordinated to the same metal ion through the 3'-bridging oxygen.

Neither of two nonbridging oxygens of phosphate moiety contacts the metal ion. Such a model of active site was also preferred by Flick et al. [5], discussing the mode of action of homing endonuclease I-PpoI. Our choice of the mechanism as presented in Fig. 1A is based upon arguments concerning the stereoselectivity of Serratia enzyme towards stereoregular oligo(nucleoside phosphorothioate)s [6,7]. Since 1995, it has been known that Serratia marcescens endonuclease is stereoselective toward oligo(nucleoside phosphorothioate)s and cleaves only internucleotide phosphorothioates of $R_{P}$ configuration (Fig. 1A; b) [6]. Although that cleavage required $10^{3}$ higher concentration of enzyme than that effective for the cleavage of isosequental PO-oligonucleotide, a further increase in enzyme concentration does not cause the cleavage of any of eleven phosphorothioate internucleotide bonds of isosequen-

\footnotetext{
*Lecture presented at the $13^{\text {th }}$ International Conference on Organic Synthesis (ICOS-13), Warsaw, Poland, 1-5 July 2000. Other presentations are published in this issue, pp. 1577-1797.

$\dagger$ Corresponding author
} 
(A)

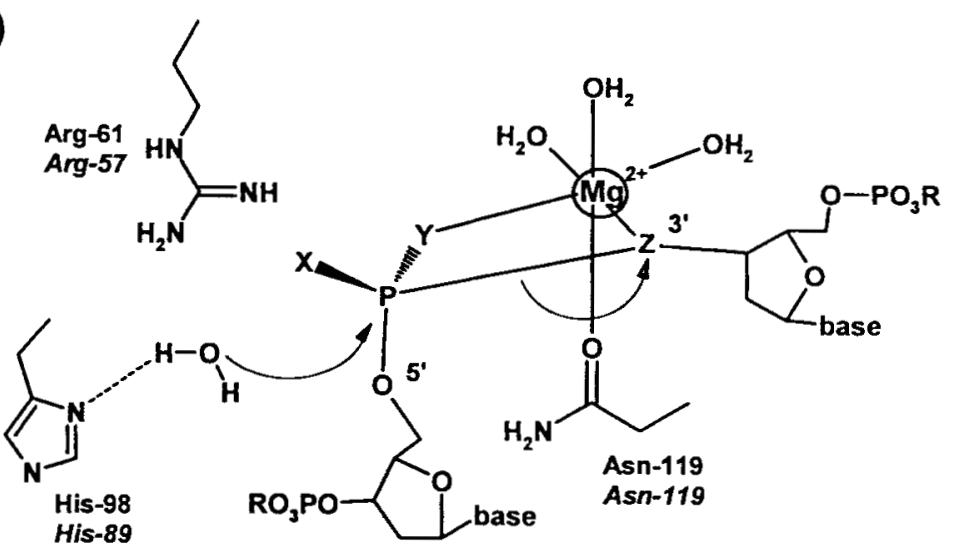

(B)
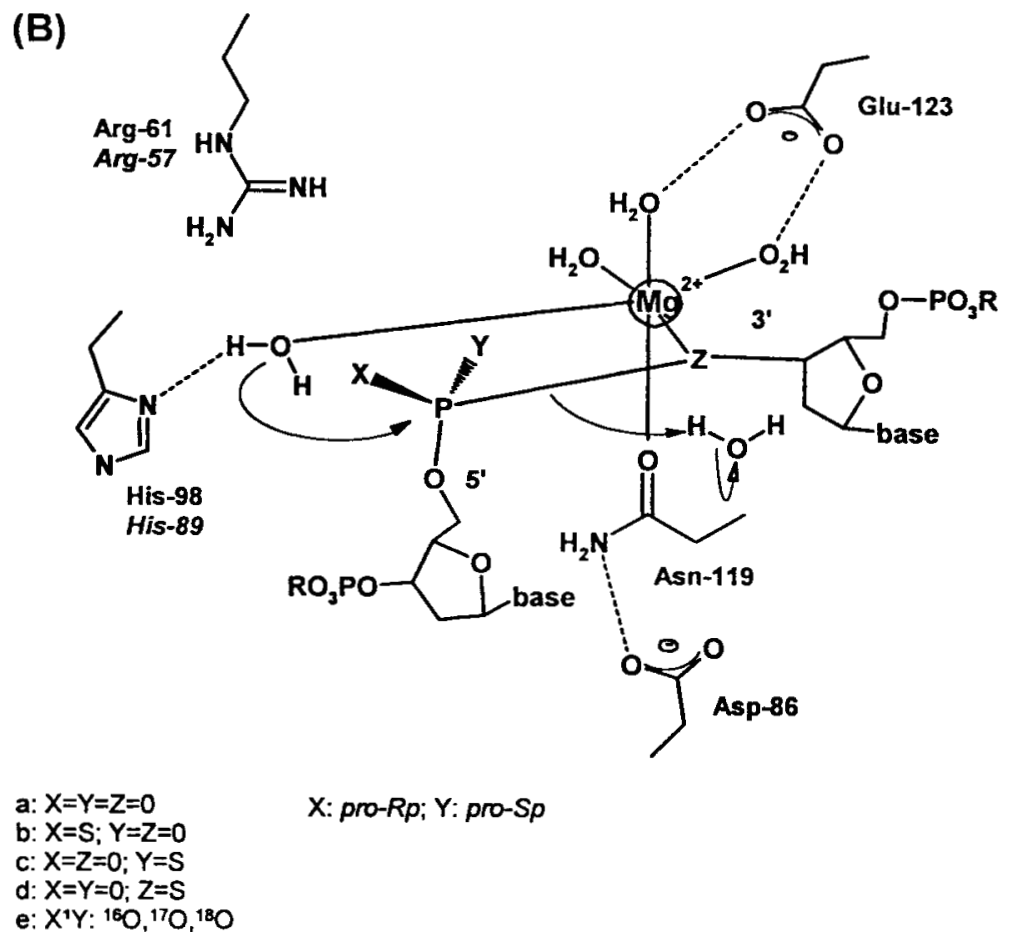

$\mathrm{X}$ : pro-Rp; Y: pro-Sp

Fig. 1 Mechanistic schemes for cleavage of internucleotide bond by Serratia and I-PpoI endonucleases (as discussed in refs. 4 and 5). Elemental (A; b, c, d) substitution of the nonbridging (X,Y) and 3'-bridging (Z) oxygen atoms by sulfur, and isotopic (A; e) replacements of the nonbridging $(\mathrm{X}, \mathrm{Y})$ oxygen atoms by its stable isotopes ${ }^{17} \mathrm{O}$ and/or ${ }^{18} \mathrm{O}$ are indicated. Single replacement of the pro- $R_{P}$-nonbridging oxygen by sulphur $(\mathrm{A} ; \mathrm{b})$ fits into the architecture of the active site, and the enzyme is able to cleave $\mathrm{P}-\mathrm{O}^{3^{\prime}}$ bond of the modified (in this way) internucleotide linkage. Replacement of the pro- $S_{P}$-nonbridging oxygen by sulphur (A; c) disturbs the fitness of components of the active site, making $S_{P}$-phosphorothiate resistant to the enzyme action. The arrangement presented in Fig. 1B does not distinguish between $\mathrm{X}$ and $\mathrm{Y}$; therefore, Fig. 1A better explains the spatial requirements and stereoselective discrimination between $R_{P^{-}}$and $S_{P^{-}}$-phosphorothioates.

tial $S_{P}$-PS-oligonucleotide. All-S $S_{P}$-PS-oligonucleotides (Fig. 1A; c) remained resistant to this enzyme, also in the presence of $\mathrm{Mn}^{2+}$ ions. Such stereodependent avidity of Serratia enzyme toward All-PS- 
oligonucleotides was confirmed for several oligo(nucleoside phosphorothioate) constructs with different base sequences and allowed the use of tandem Serratia marcescens and snake venom 3'-exonuclease as a tool for assignment of diastereomeric purity of stereodefined PS-oligos [7].

Meanwhile, Friedhoff et al. [8] reported interesting results of their stereochemical studies using pentadeoxyadenylate and its four diastereoisomeric monophosphorothioate (but each separated into pure $R_{P}$ and $S_{P}$ diastereomers) congeners with phosphorothioate function located in each internucleotide position. They concluded that modification of a single internucleotide bond of pentadeoxyriboadenylic acid with phosphorothioate causes reduction of the cleavage rate at the modified phosphodiester bond by a factor of $10^{3}$ for the $R p$ - and at least $10^{5}$ for the $S_{P^{-}}$diastereomer $\left(R_{P} / S_{P}>100\right)$. Moreover, those authors indicated, in agreement with an observation reported earlier for Eco-RI endonuclease [9], the influence of the sense of chirality of modification upon the rate of cleavage of neighboring phosphates. $S_{P}$-phosphorothioate prevented the cleavage at this group and strongly retarded cleavage of the phosphate 5 ' to the substitution, while $R_{P}$ phosphorothioate slowed down the rate of cleavage at the site of modification, but enhanced the rate of cleavage at the 5'-adjacent position [8]. These observations need to be interpreted carefully because it has been previously demonstrated that cleavage is not necessarily directly dependent on the sense of chirality of phosphorus of the phosphorothioate moiety, but can be influenced by the change in DNA conformation [10-11].

The proposed catalytic mechanism for I-PpoI/Serratia endonucleases concerns the direct contact of the pro- $S_{P}$-nonbridging oxygen and the 3'-bridging oxygen with hydrated magnesium ion [3]. Elemental replacement of any of these two oxygens by sulfur destroys the architecture of the active site, enabling the attack of a water molecule activated by the histidine residue located at the face opposite to the scissile $\mathrm{P}-\mathrm{O}^{3}$ bond (Fig. $1 \mathrm{~A}$; $\mathrm{c}-\mathrm{d}$ ). This was demonstrated by our observation of stereoselective cleavage of only $\mathrm{R}_{\mathrm{P}}$-phosphorothioates caused by Serratia enzyme, but also by the experiments described by Flick et al. [5]. Replacement of the 3'-bridging oxygen of the scissile phosphate by sulfur also prevents cleavage, albeit it renders phosphorus achiral (Fig. 1A; d). However, single elemental replacement of the pro-Rp-nonbridging oxygen by sulfur does not distort the architecture of the active site (Fig. 1A; b). Therefore, the cleavage of the $\mathrm{P}-\mathrm{O}^{3}$ bond may occur, although at a lower rate. That is not surprising in light of numerous reports from the chemistry of phosphorus emphasizing the lower rate of nucleophilic substitution at phosphorothioates, as compared with parent phosphates [12].

Taking into account the arguments presented above, the mechanism of action depicted in Fig. 1B does not fulfil the enzyme's requirement for stereoselectivity due to the poorer stabilization of pentacoordinate transition state and/or the possibility of rotation around the $\mathrm{P}-\mathrm{Z}$ bond. However, few additional comments are required. First, the arrangement of all components of the active site as presented in Fig. 1A ignores the role and involvement of the adjacent 3'-phosphate, pointed out earlier by Friedhoff (vide supra). Based on recent studies of Horton et al. [13] on inhibition of Eco-RV by deoxyribo-3'-S-phosphorothiolates the most feasible explanation would require an involvement of the another metal ion into the active site of Serratia enzyme, otherwise not discussed in X-ray studies [4]. Alternatively, the pro- $S_{p}$-oxygen of $3^{\prime}$-adjacent phosphate may be involved in the interactions with water-bound magnesium of active site, additionally stabilizing its conformational rigidity; 3 '-adjacent $R_{p}$-phosphorothioate enhances the cleavage of scissile phosphate, while $3^{\prime}$-adjacent $S_{p}$-phosphorothioate retards its cleavage [8].

Second, the observed failure of more than magnesium thiophilic $\mathrm{Mn}^{2+}$ ion to rescue the activity of the Serratia endonuclease toward All- $S_{P}$-oligo(nucleoside phosphorothioate)s [7] may argue against the direct contact between pro- $S_{P}$-oxygen and divalent metal ions. Numerous papers offer an interpretation of the failure of rescue activity of thiophilic metal ions via separation of scissile phosphate oxygen atom from metal ion by water [14] what would be contradictory to inner-sphere ligation of divalent metal ions to any position of the scissile phosphate, as postulated in Fig. 1A. But on the other hand, careful analysis of structural data obtained for uncleaved enzyme-substrate complex [2] indicates the distance $c a$. $2.6 \AA$ between each phosphate oxygens involved in the contact with magnesium ion that rather excludes the scenario of water separating these atoms. The failure of $\mathrm{Mn} 2+$ ion to rescue activi- 
ty of Serratia endonuclease toward All- $S_{P^{-}}$oligo(nucleoside phosphorothioate)s indicates that the elemental replacement of each pro- $S_{P}$-oxygen destroys the architecture of the active site due to the more bulky sulfur. Therefore, manganese ion, otherwise more bulky than magnesium ion, cannot accommodate the requirements of all components of the active site, and the lack of $\mathrm{Mn}^{2+}$ rescue effect speaks in

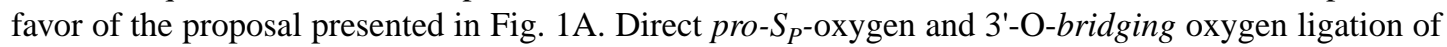
a divalent manganese ion would perturb an access and participation of Asn-119 residue as the only protein ligand bound to catalytic metal ion. Steric arguments were used for explanation of the inability of $\mathrm{Mn}^{2+}$ ion to rescue catalysis in the case of the 3',5'-exonuclease of $E$. coli DNA polymerase [15,16].

Third, the stereocontrolled replacement at each internucleotide bond, which is feasible due to the development of enzymatic [17] and chemical methods of stereocontrolled synthesis [6,18], prevents the "hoping effect" of exonucleases as was noticed earlier by Benkovic et al. [19] and also reported by others [20]. Therefore, particularly in the case of nonsequence-specific endonucleases, a sliding enzyme does not avoid any particular phosphate due to, for instance, steric effect, since the probability for that type of restriction resulting from steric requirements of a sulfur atom exists at any internucleotide bond.

However, from the more idealistic perspective, one has to admit that elemental replacement brings severe effects, since resulting molecules are always "unnatural substrates" exposed for interaction with natural enzymes, requiring effective cleavage in the presence of natural co-catalysts, such as magnesium or zinc ions.

Affinity of sulfur towards magnesium is different from that for oxygen [21]. Charge distribution at anionic phosphorothioate is also often debatable [22]. Moreover, those otherwise intelligent replacements cause the change not only in the geometry of metal coordination sphere in the active site, but also in hydration (T. James and K. Lind, personal communication). Phosphorothioate and phosphate have different affinities toward water, and also due to the slightly different pKa it may disturb local acid-base relationships, as pointed out in recent studies on the mechanism of action of ribozymes [23-24]. Therefore, an ideal solution would involve the use of stereospecifically isotopically labelled oligonucleotides, (Fig. 1A; e) as originally proposed and implemented in studies on phosphoryl transfer by Knowles et. al. [25] and Lowe et. al. [26]. Although their synthesis is feasible [27-29], improved analytical methods are needed before they can be used reliably [30,31].

\section{ACKNOWLEDGMENTS}

Presented results were financially assisted by the State Committe for Scientific Research, grant no. PBZ-KBNK005/T09/1999. W. J. S. expresses his gratitude to MITI for supporting his three-month Visiting Professorship in the Laboratory of Molecular Biology, National Institute for Advanced Interdisciplinary Research in Tsukuba.

\section{REFERENCES AND NOTES}

1. P. Friedhoff, I. Franke, G. Meiss, W. Wende, K. L. Krause, A. Pingoud. Nature Struct. Biol. 6, 112-113 (1999).

2. E. A. Galburt, B. Chevalier, W. Tang, M. S. Jurica, K. E. Flick, R. J. Monnat, B. L. Stoddart. Nature Struct. Biol. 6, 1096-1099 (1999).

3. P. Friedhoff, I. Franke, K. Krause, A. Pingoud. FEBS Letters 443, $209-214$ (1999).

4. M. D. Miller, J. Cai, K. Krause. J. Mol. Biol. 288, 975-987 (1999).

5. K. Flick, M. S. Jurica, R. J. Monnat, B. L. Stoddart. Nature 394, 96-101 (1998).

6. W. J. Stec, A. Grajkowski, A. Kobylańska, B. Karwowski, M. Koziołkiewicz, A. Okruszek, A. Wilk, P. Guga., M. Boczkowska. J. Am. Chem. Soc., 117, 12019-12029 (1995).

7. M. Koziokiewicz, A. Owczarek, E. Gendaszewska. Antisense \& Nucl. Acids Drug Dev. 9, 171-181 (1999). 
8. P. Friedhoff, G. Meiss, B. Kolmes, U. Pieper, O. Gimadutdinow, C. Urbanke, A. Pingoud. Eur. J. Biochem. 241, 572-580 (1996).

9. W. J. Stec, Zon., W. Egan, B. Stec. J. Am. Chem. Soc. 106, 6077-6079 (1984).

10. D. R. Lesser, A. Grajkowski, M. R. Kurpiewski, M. Koziołkiewicz, W. J. Stec, L. Jen-Jacobson. J. Biol. Chem. 267, 24810-24818 (1992).

11. M. R. Kurpiewski, M. Koziołkiewicz, A. Wilk, W. J. Stec, L. Jen-Jacobson. Biochemistry 35, 8846-8854 (1996).

12. D. Barton and W. D. Ollis. Comprehensive Organic Chemistry, Vol. 2, pp. 1257-1300, Pergamon;

D. Herschlag, J. A. Piccirilli, T. R. Cech. Biochemistry 30, 4844-4854 (1991).

13. N. C. Horton, B. A. Connolly, J. J. Perona. J. Am. Chem. Soc., 122, 3314-3324 (2000).

14. N. C. Horton, K. J. Newberry, J.J. Perona. Proc. Natl. Acad. Sci. U.S.A., 95, 13489-13494 (1998).

15. C. A. Brautigam and T. A. Steitz. J. Mol. Biol. 277, 363-377 (1998);

16. C. A. Brautigam, S. Sun, J. A. Piccirilli, T. A. Steitz. Biochemistry, 38, 696-704 (1999).

17. J. G. Hacia, P. B. Dervan, B. J. Wold. Biochemistry, 33, 6192-6200 (1994).

18. W. J. Stec, A. Grajkowski, M. Koziołkiewicz, B. Uznański. Nucleic Acid Res. 19, 5883-5888 (1991).

19. F. R. Bryant and S. J. Benkovic. Biochemistry 18, 2825-2828 (1979).

20. W. J. Stec and G. Zon. In Natural Product Chemistry 1984, R. I. Zalewski and J. J. Skolik (Eds.), pp. 495-510, Elsevier (1985).

21. R. K. O. Sigel, B. Song, H. Sigel. J. Am. Chem. Soc. 119, 744-755 (1997).

22. P. A. Frey and R. D. Sammons. Science 228, 541-547 (1985).

23. D.-M. Zhou and K. Taira. Chem. Rev. 98, 991-1026 (1998).

24. M. Warashina, Y. Takagi, W. J. Stec, K. Taira. Current Opinion in Biotechn. 11, 354-362 (2000).

25. S. L. Buchwald, D. Hansen, A. Hasset, L. R. Knowles. Methods in Enzymology, Vol. 87, pp. 279-301, Academic Press (1982).

26. G. Lowe. Accounts Chem. Res. 16, 244-251 (1983).

27. For exhaustive review, see P. A. Frey. Advances in Enzymology, Vol. 62, pp. 119-201, Wiley, New York (1989).

28. Labelling of the nucleoside 3'-O(2-thiono-1.3.2-oxathiaphospholanes) allows for the stereocontrolled synthesis of $\left[{ }^{16} \mathrm{O},{ }^{18} \mathrm{O}\right]$-isotopomeric oligonucleotides; P. Guga, K. Domański, W. J. Stec. Angew. Chem. 40, in press (2001).

29. W. J. Stec, B. Karwowski, M. Boczkowska, P. Guga, M. Koziołkiewicz, M. Sochacki, M. Wieczorek, M. Błaszczyk. J. Am. Chem. Soc. 120, 7156-7167 (1998).

30. M. H. O'Leary. Annu. Rev. Biochem. 58, 377-401 (1989).

31. V. Cepus, A. J. Scheidig, R. S. Goody, K. Gerwert. Biochemistry 37, 10263-10271 (1998). 